\title{
Effects of vibrotactile-enhanced music- based intervention on sensorimotor control capacity in the hand of an aging brain: a pilot feasibility randomized crossover trial
}

Hsiu-Yun Hsu ${ }^{1,2,3}$, Che-Wei Lin ${ }^{3,4}$, Yu-Ching Lin ${ }^{1,5}$, Po-Ting Wu' ${ }^{6}$, Hirokazu Kato ${ }^{7}$, Fong-Chin Su ${ }^{3,4}$ and Li-Chieh $\mathrm{KuO}^{2,3^{*}}$

\begin{abstract}
Background: Music-based interventions (MBI), using music as a therapeutic medium, has been utilized as a promising strategy for motor relearning and shaping. However, currently, MBI with active performance training is restricted to being extensively applied for patients with various levels of defects in fine motor skills and cognitive functions. Therefore, the integration of vibrotactile stimulation with MBI has been adopted as a motor training strategy intended to enhance motor learning through use of vibration stimuli. The current study was designed to investigate differences in the sensorimotor performance of older adults' hands under baseline, a single session of active MBI, and vibrotactileenriched $\mathrm{MBI}$ conditions.
\end{abstract}

Methods: Thirty healthy older adults were recruited and randomized to receive either the single session of 30-min of vibrotactile-enriched $\mathrm{MBI}$ or 30-min of active MBI at the beginning of the experiment. After a one-week washout period, they switched their treatment programs and then were assessed to study the training effects of both approaches through measuring precision pinch performance, hand function, and sensory status.

Results: The results of the Pinch-Holding-Up Activity test revealed a statistically significant difference in the $F_{\text {peak }}$ parameter $\left(F=14.37, p<0.001, \eta^{2}{ }_{p}=0.507\right)$ under the vibrotactile-enriched $\mathrm{MBI}$ condition compared to the baseline and active $\mathrm{MBI}$ conditions. In addition, significant beneficial effects were found on the results of the barognosis $\left(F=19.126, p<0.001, \eta_{p}^{2}=0.577\right)$ and roughness differentiation subtests $\left(F=15.036, p<0.001, \eta_{p}^{2}=0.518\right)$ in the Manual Tactile Test for the participants in the vibrotactile-enriched MBI group. In addition, the participants under both the active $\mathrm{MBI}$ and vibrotactile-enriched $\mathrm{MBI}$ conditions exhibited better performance in the three subtests of the Purdue Pegboard Test as compared to under the baseline condition $(p<0.016)$.

Conclusions: The findings indicated that vibrotactile-enriched MBI potentially improves the precision pinch performance of hands in healthy older adults. In addition, the add-on effect of vibrotactile stimulation to the $\mathrm{MBI}$ condition provides beneficial effects on the sensory functions of the upper extremities.

Trial registration: NCT04802564.

\footnotetext{
*Correspondence: jkkuo@mail.ncku.edu.tw

${ }^{2}$ Department of Occupational Therapy, College of Medicine, National

Cheng Kung University, Tainan, Taiwan

Full list of author information is available at the end of the article
} original author(s) and the source, provide a link to the Creative Commons licence, and indicate if changes were made. The images or other third party material in this article are included in the article's Creative Commons licence, unless indicated otherwise in a credit line to the material. If material is not included in the article's Creative Commons licence and your intended use is not permitted by statutory regulation or exceeds the permitted use, you will need to obtain permission directly from the copyright holder. To view a copy of this licence, visit http://creativecommons.org/licenses/by/4.0/. The Creative Commons Public Domain Dedication waiver (http://creativeco mmons.org/publicdomain/zero/1.0/) applies to the data made available in this article, unless otherwise stated in a credit line to the data. 
Date of registration: 15/03/2021.

The first posted date: 17/03/2021.

Keywords: Music-based intervention, Haptic, Sensorimotor, Hand function

\section{Background}

The number and percentage of the older population is rapidly increasing worldwide. Aging declines skill performance related to physiological changes in skeletal muscles [1] as well as reduced functional integration of the sensory-motor system [2]. It is worth noting that manual dexterity of the hand has long been known as an early indicator of age-related functional decline [3]. A previous study revealed that aging strongly impacts motor performance such as fingertip force modulation requiring online sensory input [4]. Specifically, the population with old age might usually be reported with their impairment of motor adjustments in response to the environmental perturbation and task demands [5] which might impact on the execution of daily living activities. Thus, activities, such as music for maintaining and even promoting the sensorimotor control capabilities of an aging brain [6] should be given attention.

Sensory-augmented therapy has been proposed as a helpful adjunctive strategy that can be used to enhance the effects of motor retraining when integrated with conventional rehabilitation programs for patients with sensorimotor deficits $[7,8]$. Vibrotactile sensation caused by a mechanical stimulus characterized by an oscillating motion has been reported to enhance muscular function as well as neuronal activity [9]. A recent study revealed that the activity of the primary sensorimotor area is increased significantly during processing of highfrequency vibrotactile information [10]. In addition, light touch sensation has been shown to be improved in patients' hands when performing a specific task with vibrotactile stimuli on the dorsum of the wrist [11]. This effect may be due to the enhancement of the excitability of sensory neurons through interneuronal connections during task execution [12].

In addition to vibrotactile stimuli, sensory-based interventions to improve hand function also include visual and auditory strategies. Music-based intervention (MBI), using music as a therapeutic medium, has been utilized as a promising strategy of neuro-rehabilitation in the last decade [13]. The delivery mode of MBI includes the following main types: active, receptive and combined active and receptive intervention [14]. The information providing by music auditory stimuli induces plastic changes in the brain through the effects of auditory-motor entrainment and sensorimotor synchronization for patients with neurological impairment $[15,16]$. The active mode of MBI has been reported to be effective in restoration of motor skills of upper limb [17]. Playing keyboards and drums have been reported as the most popular training regimes used to improve fine motor and gross motor coordination, respectively [18]. MBI with active performance is currently considered to be a practical framework based on neuroscience used for motor relearning and shaping, audio-motor coupling, and evoking emotional effects since individuals receive auditory melody and rhythmic feedback [19]. Recent evidence revealed that the finger dexterity of subacute stroke patients was improved through providing diverse sensory rewards for sequential finger movements based on keyboard playing programs [20]. Specifically, MBI with active performance increased the activity and connectivity between the auditory and motor cortical regions by providing individuals with auditory feedback for errors and real-time movement adjustments, which consequently boosted motor recovery of the upper limbs of stroke patients [21]. In addition to patients with neurological deficits, a recent study also revealed that active music therapy has effects on improving upper limb muscle power in community dwelling older adults [22]. However, the effects of MBI with active performance on the sensorimotor control capacity of an aging hand closely related to lifestyle and living quality are as yet not well known.

MBI with active performance mainly focuses on movement relearning and shaping through training in the use of musical instruments or specifically-designed electronic devices. Despite the fact that positive findings have been reported [23, 24], the current active MBI protocol has limitations related to widespread application in patients with different or varied levels of fine motor skills and cognitive functions. Therefore, musical haptics, a new concept of adopting vibrotactile stimulation in MBI, which convey the music information through skin of the finger pulps is suitable to enrich the musical listening experience [25] and improve significantly on sensorimotor function [26]. In addition, vibrotactile stimulation has been reported to enhance the performance of the affected arm in stabilization and reaching tasks given to patients suffering from neurological diseases [27]. However, the difference in the training effects between the active MBI and enriched MBI with vibrotactile stimulation on hand functions has not been appropriately investigated. Thus, the purpose of this study was to analyze the difference in the effects on sensorimotor control capacity in the 
hand of an aging brain across baseline, active MBI, and vibrotactile-enriched MBI conditions. For this purpose, a vibrotactile-enriched interfaces for MBI system was designed to assist with performing MBI in this study. We hypothesized that both one-session of active MBI, and vibrotactile-enriched MBI treatment paradigm may lead to improvements in sensorimotor performance of a hand. The significance of this study was a vibrotactile-enriched MBI system providing multi-sensory experience has been developed and the feasibility of clinical practice has been tested. The findings of this study would highlight the benefit of active and vibrotactile-enriched MBI on sensorimotor performance of a hand.

\section{Methods}

\section{Study design}

An assessor blinded, randomized controlled, crossover design was used in the study. The participants were randomly assigned in a 1:1 ratio into either a one-session active MBI group or a vibrotactile-enriched MBI group. After a one-week washout period, they switched treatment programs. The assessments were conducted with the time of pre-treatment as the baseline $\left(\mathrm{T}_{\mathrm{b}}\right)$, immediately followed by one-session of the active MBI $\left(\mathrm{T}_{\mathrm{a}}\right)$ and vibrotactile-enriched MBI $\left(\mathrm{T}_{\mathrm{v}}\right)$. Two assessors who underwent in-person training on assessments performing were blinded to the participant's condition. One was an occupational therapist (for the Semmes-Weinstein monofilament and Purdue Pegboard Test measures) and the other one was a technician (for the pinch-holdingup activity and manual tactile test measure). The data were collected at the department of physical medicine and rehabilitation in a medical center setting in southern Taiwan.

\section{Participants}

Thirty community dwelling healthy older adults were recruited based on an estimation of the effects obtained regarding hand performance using a previous sensory augmented rehabilitation program estimated with a 2-tailed alpha of 0.05 and a power of 0.95 [28]. The inclusion criteria for all the participants in this research group were as follows: (1) right-handed; (2) age ranging from 55 to 85 ; (3) no history of neurological or psychiatric illness, no severe vision or hearing loss, no abnormalities in the upper extremities, (4) the capacity to perform and maintain a pinch task with the thumb and index finger while lifting an object, (5) no previous musical instrument education, and (6) a score of 24 or more on the Mini Mental State Examination. Participants with difficulty following instructions, diagnosed with neuro-musculoskeletal disorders, or having a poor attention span were excluded. Prior to participation, each participant was asked to sign a consent form after being informed of the objectives and the related research procedures. Fifteen male and 15 female older adults between the ages of 55 and 85 (65.7 \pm 5.6 years old) were recruited in this study. The participants were with an average educational level of $11.8 \pm 3.2$ years. All participants completed the required outcomes measurements for the three conditions.

\section{Randomization and allocation concealment}

The participants were randomly allocated to either active MBI or vibrotactile-enriched MBI condition first by a computer-generated random number sealed in opaque envelopes. The therapist opened the envelope and found the treatment to be conducted in this participant.

\section{Equipment}

The vibrotactile-enriched interfaces for MBI system (Fig. 1) is a custom-made training apparatus composed of three distinct parts: (1) A laptop computer: This computer produces music output integrated with visual information in the form of a color bar moving on the screen display corresponding with the rhythmic elements of the music. (2) Haptic feedback component: To establish the haptic interface, an Arduino UNO microcontroller board was used as a microprocessor, which controlled and worked with five coin-shaped micro vibration motors (Model \#1027, TAIWAINIOT ${ }^{\mathrm{TM}}$, Taiwan) to achieve the function of providing vibrotactile feedback in the system. The motor was driven at a voltage of $5 \mathrm{~V}_{\mathrm{DC}}$ (direct current) with sinusoidal vibration applied to the fingertip at a frequency of approximately $200 \mathrm{~Hz}$. The pulp of each digit was securely positioned in a specially designed 3D printing base as a reinforced structure with Velcro-fastenings. (3) Image classifier: The artificial intelligence deep residual network (ResNet50) was used for precise image

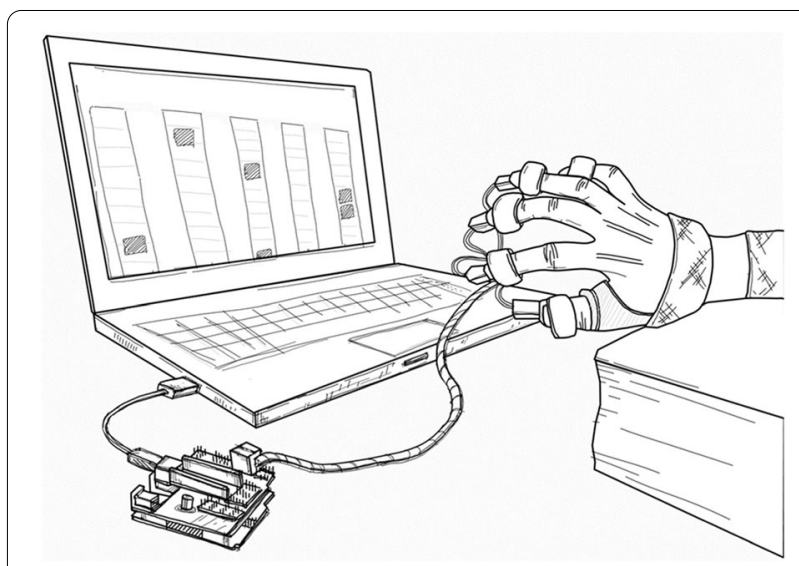

Fig. 1 Illustration of the vibrotactile-enriched music-based intervention system 
recognition and the integration of the visual information from the moving color bar and vibrotactile stimuli in a timely manner.

\section{Intervention}

Two training regimes, the active MBI and vibrotactileenriched MBI regimes, based on a crossover study design with a one-week washout phase were applied for all the participants. The details of intervention delivering in this study was based on a reporting guideline of musicbased interventions [29]. Ten familiar musical compositions with the tempo ranging between 49 and 100 beats/ minute from the Beatles, consisting simple melody with periodic cycles, were pre-selected by the investigator as the materials for both the active MBI and vibrotactileenriched MBI which provided participants with melodies and rhythms. Music was delivered by speakers with a volume controlled lower then $65 \mathrm{~dB}$ by an occupational therapist. The active MBI is aimed toward improving the participants' fine motor skills through a 30-min "active practice" session. The participants were instructed to play music by pressing the keyboard with the corresponding digits guided by auditory and visual feedback displayed as a moving color-bar on the computer screen. The hand was allowed to perform different kinds of pressing activities, such as single and multiple digits pressing in a specific rhythm and melody on a computer keyboard. The difficulty level could be adjusted for each participant to meet the motor skill level. In the vibrotactile-enriched MBI, the system provided 30-min of vibrotactile stimuli synchronized with audio and visual information to each digit via small, coin-shaped vibration motors fitted on the pulps of the digits while the music was played. Different from the active MBI, the participants receiving the vibrotactile-enriched MBI were asked to receive multiple sensory information, including visual, auditory, and vibrotactile stimuli, but did not actively perform piano key pressing tasks. The stimulus sequences were same in both the active and vibrotactile-enriched MBI conditions.

\section{Outcome measures}

\section{Primary outcome measures}

The pinch-holding-up activity (PHUA) test is a taskbased assessment with high test-retest reliability (intraclass correlation coefficient ranged from 0.84 to 0.96 ) [30] was used for detecting the sensorimotor control of a hand with functional perspective. A $480 \mathrm{~g}$-weight pinch apparatus with two load cells and one accelerometer used to detect the pinch force exertion of a hand and acceleration of the apparatus in space, respectively, were used to examine the pinch and load force coupling related to lifting performance of the upper extremity. The testing procedures comprised two phases, a holding phase and a lifting phase. The participants were instructed to pinch and lift the apparatus to a $5 \mathrm{~cm}$ height above the table and then asked to hold the device in this position for $5 \mathrm{~s}$ (holding phase). Afterwards, the pinch-apparatus was lifted from $5 \mathrm{~cm}$ to the height of $30 \mathrm{~cm}$ (lifting phase) above the table and then slowly lowered to the initial position. The total duration of data collection was approximately $15 \mathrm{~s}$, where the maximum upward acceleration of the apparatus and peak pinch force exerted by the digits during the lifting phase of the test were recorded. Test procedures were repeated three times with between-trial resting interval of $1 \mathrm{~min}$ ute. The peak pinch force during the lifting phase was defined as $\mathrm{FP}_{\text {peak }}$, and the maximum load of the object was defined as $\mathrm{FL}_{\max }$. $\mathrm{FR}_{\text {peak }}$, the ratio between $\mathrm{FP}_{\text {peak }}$ and $\mathrm{FL}_{\max }$, indicated the ability of a hand to adjust to the pinch force related to changes in the inertial load of the lifted object. The $\mathrm{FR}_{\text {peak }}$ for young healthy adult ranged between 1.77 and 1.98 [30]. $\mathrm{FR}_{\text {peak }}$ is a sensitive parameter used to assess the sensorimotor control of the hand in the form of a dynamic coordination model [31]. An elevated $F R_{\text {peak }}$ indicated insufficient ability of sensorimotor control in a hand [32].

\section{Secondary outcome measures}

The manual tactile test (MTT) is a timed evaluation tool used to determine the discriminative sensation involved in active exploration of a hand with reported reliability, accuracy, and validity [33]. Three subtests in the MTT, barognosis, roughness differentiation, and stereognosis, were conducted for evaluating the hand perceptions of a participant related to distinguishing object characteristics - weight, roughness, and shape, respectively, with active touch while blindfolded. The test procedures for each subtest were repeated three times for each hand. The average time required to perform each test was calculated to arrive at the final score of that test. The lower the score obtained, the inferior of his discriminating sensibility.

The Semmes-Weinstein monofilament (SWM) test is the most responsive touch-pressure sensory test [34]. Higher score of the SWM test represented the poorer tactile sensation. It also has reliability and specificity for identifying a loss of protective sensation in seniors [35]. A full set of SWM contains 20 monofilaments, and each monofilament is labeled with a numerical marking, which is a log to the base ten of force in tenths of a milligram. When conducting the SWM test, the filaments are applied perpendicular to the pulp of each digit with a constant force onto the skin area for 1-1.5 s.

The Purdue Pegboard Test (PPT) is a test of the unimanual and bimanual dexterity of the hands. It has been demonstrated to have high testing validity and 
reliability [36]. The test was carried out using a procedure in which pins are inserted into small holes with the dominant hand and both hands simultaneously in $30 \mathrm{~s}$, as well as an assembly task that was performed for $60 \mathrm{~s}$. Higher score is indicative of better finger dexterity.

\section{Statistical analysis}

SPSS 19.0 for Windows (Statistical Package for Social Sciences Inc. Chicago, IL, USA) was used for the statistical analyses. The descriptive statistics were used to describe the means and standard deviations of the characteristics data and outcome measurements, including the results of the PHUA, MTT, SWM, and PPT. Normality in the data distribution was examined with a Shapiro-Wilk's test $(p \geq 0.05)$. All variables fulfilled the normality criteria; therefore, the repeated measure ANOVA was used to determine if there are difference across the following conditions, the baseline, one-session of the active MBI, and one-session of vibrotactile-enriched MBI. Statistical significance was set at $p<0.05$. For each repeated measures ANOVA, the partial eta squared $\left(\eta_{\mathrm{p}}^{2}\right)$ was presented as a measure of effect size. The Bonferroni post hoc test was used to examine whether any differences existed between the different conditions as the main ANOVA is significant. After the Bonferroni correction, the statistical threshold was adjusted to $p<0.016$.

\section{Results}

Table 1 summarizes the descriptive statistics and the results of the repeated measure ANOVA for the precision pinch performance, hand function, and sensory status under the three conditions of interest. A statistically significant difference was detected for the $\mathrm{FR}_{\text {peak }}$ parameter $\left(\mathrm{F}=14.37, p<0.001, \eta_{\mathrm{p}}^{2}=0.507\right)$ and $\mathrm{FP}_{\text {peak }}(\mathrm{F}=7.295$, $\left.p=.003, \eta_{\mathrm{p}}^{2}=0.343\right)$ using the PHUA test, across all three conditions. Based on the post-hoc examination, the participants under the vibrotactile-enriched MBI condition had better capacity to modulate their pinch force according to the fluctuated load of the lifted object in terms of their dynamic pinch-lifting performance compared to the baseline and active MBI conditions (Table 1). However, there were no statistically significant differences for the precision pinch performance between the baseline and active MBI $(p=0.304$ and $p=0.165$, respectively, for $\mathrm{FR}_{\text {peak }}$ and $\mathrm{FP}_{\text {peak }}$ ).

In terms of sensory function, there was a statistically significant difference in the results of the MTT and SWM test for the participants across all three conditions (Table 1). The SWM results under the vibrotactileenriched MBI condition was better than that under the baseline condition $(p=0.010, p=0.001$, and $p=0.004$, respectively, for the thumb, index finger, and little finger). However, the SWM results under the active MBI condition revealed no statistically significant differences compared to the baseline condition $(p=0.069, p=0.038$ and $p=0.063$, respectively, for the thumb, index finger,

Table 1 The descriptive statistics and the results of the repeated measure ANOVA used to determine the precision pinch performance, hand function, and sensory status under the three conditions

\begin{tabular}{|c|c|c|c|c|c|c|c|}
\hline & & \multicolumn{3}{|l|}{ Conditions } & \multicolumn{3}{|c|}{ Difference across conditions } \\
\hline & & Baseline & Active MBI & $\begin{array}{l}\text { Vibortactile- } \\
\text { enriched MBI }\end{array}$ & $\mathrm{F}$ & $p$ & $\eta_{p}^{2}$ \\
\hline \multirow[t]{2}{*}{ PHUA } & $F P_{\text {peak }}(N)$ & $13.19 \pm 1.54$ & $12.91 \pm 1.15^{\ddagger}$ & $12.40 \pm 1.01^{\dagger \neq}$ & 7.295 & .003 & .343 \\
\hline & $F R_{\text {peak }}$ & $2.98 \pm .33$ & $2.94 \pm .30^{\ddagger}$ & $2.76 \pm .23^{\dagger \neq}$ & 14.370 & $<0.001$ & .507 \\
\hline \multirow[t]{3}{*}{ MTT (seconds) } & Barognosis & $3.18 \pm .10$ & $3.10 \pm .09^{\ddagger}$ & $2.81 \pm .09^{\dagger \neq}$ & 19.126 & $<0.001$ & .577 \\
\hline & Roughness differentiation & $31.12 \pm 6.40$ & $28.56 \pm 4.13^{* \neq}$ & $27.49 \pm 3.60^{\dagger \neq}$ & 15.036 & $<0.001$ & .518 \\
\hline & Stereognosis & $24.86 \pm 2.65$ & $23.46 \pm 2.71^{*}$ & $22.40 \pm 4.39^{\dagger}$ & 9.057 & 0.001 & .393 \\
\hline \multirow[t]{3}{*}{ SWM test (gm) } & Thumb & $.181 \pm .211$ & $.156 \pm .206$ & $.130 \pm .205^{\dagger}$ & 4.389 & .022 & .239 \\
\hline & Index finger & $.161 \pm .170$ & $.124 \pm .153^{* \neq}$ & $.083 \pm .111^{\dagger \neq}$ & 6.373 & .005 & .313 \\
\hline & Little finger & $.123 \pm .143$ & $.093 \pm .109^{\ddagger}$ & $.060 \pm .073^{\dagger \neq}$ & 4.700 & .017 & .251 \\
\hline \multirow[t]{3}{*}{ PPT } & Pin insertion- $\mathrm{DH}$ & $14.1 \pm .4$ & $15.0 \pm .3^{*}$ & $15.2 \pm .3^{\dagger}$ & 8.454 & 0.001 & .377 \\
\hline & Pin insertion- $\mathrm{BH}$ & $11.3 \pm 1.6$ & $12.1 \pm 1.2^{*}$ & $11.9 \pm 1.4^{\dagger}$ & .11 .932 & $<0.001$ & .460 \\
\hline & Assembly & $31.8 \pm 5.9$ & $35.6 \pm 5.6^{*}$ & $34.6 \pm 5.2^{\dagger}$ & 18.458 & $<0.001$ & .568 \\
\hline
\end{tabular}

A repeated measure ANOVA was used to compare the effects of the different interventions. The level of significance was set at $p<0.05$. A post hoc test was used to examine whether any differences existed among the different conditions. After the Bonferroni correction, the statistical threshold was adjusted to $p<0.016$

${ }^{*}$ : Significant difference between Baseline and Active MBI. ${ }^{\dagger}$ : Significant difference between Baseline and Vibortactile-enriched MBI. ${ }^{\ddagger}$ : Significant difference between Active $\mathrm{MBI}$ and Vibortactile-enriched MBI

$F R_{\text {peak }}$ Force ratio $\left(\mathrm{FP}_{\text {peak }}: \mathrm{FL}_{\text {max }}\right), F P_{\text {peak }}$ maximum pinch force during the lifting phase, MTT Manual tactile test, SWM test Semmes-Weinstein monofilament test, PPT Purdue Pegboard Test, $\mathrm{DH}$ Dominant hand, $\mathrm{BH}$ Both hands 
and little finger). The difference in the results of SWM between the vibrotactile-enriched MBI condition and active MBI condition was not statistically significant for the thumb, index finger and little finger $(p>0.016)$. Similar to the SWM results, the sensory assessment evaluation using three MTT subtests under the vibrotactile-enriched MBI condition revealed statistically better performance compared to the baseline condition $(p<0.001, p<0.001$ and $p=0.002$, respectively, for barognosis, roughness differentiation, and stereognosis). In addition, compared to the active MBI, the participants spent relatively less time completing the barognosis $(p<0.001)$ and roughness differentiation $(p=0.014)$ subtests under the vibrotactile-enriched MBI condition. Different from the SWM results, better results were found in the roughness differentiation subtest $(p=0.006)$ and stereognosis $(p=0.001)$ following the active MBI as compared to the baseline condition; however, there was lack of statistical difference in the results for barognosis between the baseline and active MBI conditions $(p=0.077)$.

The results of the pin insertion subtests using the dominant hand, both hands, and the PPT assembly task revealed statistically significant differences across the three conditions (Table 1). The participants in both the active MBI and vibrotactile-enriched MBI conditions had faster performance in the three PPT subtests as compared to under the baseline condition.

\section{Discussion}

The aim of the present study was to evaluate the differences in the effects on the sensorimotor control capacity of a hand across conditions including baseline, active MBI, and vibrotactile-enriched MBI conditions. A vibrotactile-enriched interfaces for MBI system suitable for sensorimotor control training was developed in this study. The findings partially supported the premise that the vibrotactile-enriched MBI would enhance the sensorimotor performance of a hand in healthy older adults. Participants in the vibrotactile-enriched MBI group exhibited better performance in a pinch-holding task that required online sensory feedback, as compared to those in the active MBI and the baseline groups. In addition, the results also showed that there were better effects of the vibrotactile-enriched MBI on improving sensory functions related to barognosis and roughness differentiation, as compared to those under the baseline and active MBI conditions. This result revealed an immediate effect of a single-session vibrotactile-enriched MBI on the sensorimotor performance of healthy older adults.

The $\mathrm{FR}_{\text {peak }}$ under the baseline condition in the current study was higher when compared with data obtained from young subjects [30]. A higher pinch-to-load force ratio, which indicated inaccurate pinch force modulation to the momentum-induced load changes [37], might have been due to the decline in the sensorimotor functioning of the hands. In the case of healthy participants, pinch force predictions and adjustments during execution of a functional task is an automatic motor response corresponding to the dynamics of arm movement [38]; however, recent evidence suggested that sensory deficits of a hand appear to remarkably decrease the capacity of momentary motor adjustment [39] because task-based sensorimotor processing depends on not only a feed-forward control mechanism but also on peripheral sensory events [40]. Since aging leads to slowing of sensorimotor functions [41], an intervention program involving the use of passive sensory stimulation drives plastic reorganizational changes in the sensorimotor cortex based on the Hebbian forms of plasticity [42], which promotes precision pinch performance in the hands of healthy seniors. The better performance in precision pinch performance when receiving the vibrotactile-enriched MBI intervention was supported by recent studies based on sensory augmentation systems used to explain the potential mechanisms of sensory restoration, sensorimotor integration, and substitution in motor control $[43,44]$. That is, integration of afferent vibration signals in the form of haptic feedback in hand therapy contributes to enhancing hand performance [45].

In addition, the results of the SWM test and MTT test obtained under the baseline condition in the older adults examined in the present study revealed lower sensitivity in both the touch threshold [46] and discriminative sensory function [33]. The vibrotactile-enriched MBI had superior training effects on the results of the SWM test and the MTT barognosis subtest. Compared to the active MBI, the sensitivity related to both tactile and proprioceptive sensation increased through activation of sensory receptors during the transmission of vibrotactile stimulation to the finger tips, which enhanced sensory restoration in the participants receiving the vibrotactile-enriched $\mathrm{MBI}$ intervention. A recent report indicated that the vibrotactile component of the haptic feedback that occurs when playing a musical instrument leads to increased quality of hand perceptions [47]. Due to central mechanisms, vibrotactile stimulation on the fingertips results in not only activating cutaneous mechanoreceptors, but it also enhances the mechanical coupling between the contacted skin, tendons, and bones, which significantly improves the of touch-pressure threshold, as tested by Semmes-Weinstein monofilament [48] and the capacity of active force perception as measured when participants manipulate objects [49].

The active MBI in this study also presented some training effects on improving hand functions, which might 
concur with the findings of a previous review report regarding MST training used to enhance the motor functions of stroke survivors [50]. Active MBI provides visual and auditory information intended to guide the temporal and spatial organization of sequential motor responses based on rhythmic keyboard pressing and enhances the coordinated actions of the hand [51]. Furthermore, the predictability of motor sequences serves as a facilitative factor for motor control based on a cognitive processing mechanism that occurs during the preparation and execution of movements such as keyboard pressing during active MBI [52]. Therefore, music-based paradigms have been suggested to be an effective strategy for motor learning and rehabilitation. Also, the results of the roughness differentiation and stereognosis after the active MBI revealed statistically significant changes compared to the baseline condition in the current study, which might have been correlated with the enrichment of the sensorimotor network through functional motor training in a multisensory environment [53].

This may be the first study examining the effects of an intervention using a music-based intervention with multiple sensory feedback on sensorimotor performance of the hands of healthy senior participants. This strengths of the current pilot study include the potential training device innovation and comprehension of its feasibility for future clinical application. A novel vibrotactile-enriched MBI system provided synchronized auditory, visual and vibrotactile stimuli related to music characteristics of melody and rhythm was proposed in this study. The present study established a vibrotactile-enriched MBI system acting as a sensory augmentation approach to dealing with motor control capabilities in the hand of an aging brain. It is noteworthy that better training results in precision pinch performance, hand function, and sensory function of healthy older adults have been obtained for participants even when only receiving the vibrotactileenriched MBI intervention once comparing with receiving the active MBI. Also, both the vibrotactile-enriched MBI and active MBI interventions had beneficial effects on hand performance and sensory function compared to the baseline condition. The obtained results support the feasibility of clinical application of a sensory augmented MBI. However, several limitations exist in the present study. First of all, the protocol only provided immediate outcome measures, which make it impossible to clearly understand the delayed effects on the sensorimotor performance that occurred in both the vibrotactile-enriched $\mathrm{MBI}$ and active MBI interventions. The second limitation was lack of assessment done at the end of the washout period to ensure a true washout effect of the previous treatment. The third limitation was that the design of current study was only a single session intervention, which did not permit us to observe effects of a longer training period of music-supported therapy on the sensorimotor performance of hands. In spite of these limitations, the results indeed have merit related to supporting future studies investigating the impacts of vibrotactile-enriched MBI and active MBI on hand sensorimotor performance or sensory functioning in participants with marked sensorimotor deficits. However, the questionnaire used to measure the response regarding sensory information perceiving and reality of key pressing for MBI experiences in the two conditions needs be conducted further.

\section{Conclusions}

In summary, the pilot study supports the feasibility of using vibrotactile-enriched MBI for ameliorating sensorimotor performance in the hands of healthy older adults. Future studies are needed in order to optimize the training protocol of music-supported therapy by examining the effects of intervention intensity or time periods required for executing vibrotactile-enriched MBI and active MBI training in individuals with age-related sensorimotor deficits or neurological impairments.

\begin{abstract}
Abbreviations
MBI: Music-based intervention; PHUA: Pinch-holding-up activity; FP peak: $^{\text {Peak }}$ pinch force; $\mathrm{FL}_{\text {max }}$ : Maximum load force; $\mathrm{FR}_{\text {peak }}$ : Ratio between peak pinch force and maximum load force; MTT: Manual tactile test; SWM: SemmesWeinstein monofilament; PPT: Purdue Pegboard Test; SPSS: Statistical Package for Social Sciences; ANOVA: Analysis of variance.
\end{abstract}

\section{Acknowledgements}

Special thanks to Mr. Ze-Shiou Fang and Ms. Yu-Ting Wang for their kind assistance with participant recruitment and data collection.

\section{Authors' contributions}

$\mathrm{HYH}, \mathrm{FCS}$ and LCK were the main contributors in the determination of unmet clinical needs, the study design, and data collection. HYH, CWL, FCS, HK and LCK carried out the development of the Vibrotactile-enhanced Music-based Intervention system and technical problem solving for the system. $\mathrm{HYH}, \mathrm{YCL}$, PTW and LCK participated in the registration, screening, and examinations of the participants. HYH, PTW, and LCK participated in the data interpretation, analysis, and interpretations. HYH, CWL and LCK helped to draft the manuscript. All authors read and approved the content and format of the final manuscript.

\section{Funding}

This research work was financially supported by the Ministry of Science and Technology of TAIWAN under grant no.: MOST 109-2314-B-006-030 -MY3. This work was also financially supported by the Medical Device Innovation Center, National Cheng Kung University from the Featured Areas Research Center Program within the framework of the Higher Education Sprout Project by the Ministry of Education (MOE) in Taiwan.

Availability of data and materials Not Applicable.

\section{Declarations}

Ethics approval and consent to participate

Ethical approval: All procedures performed in studies involving human participants were in accordance with the ethical standards of the institutional and/ 
or national research committee and with the 1964 Helsinki declaration and its later amendments or comparable ethical standards. The procedures and consent form were approved by the Institutional Review Board at National Cheng Kung University Hospital.

Informed consent: Informed consent was obtained from all individual participants included in the study.

\section{Consent for publication}

Not Applicable.

\section{Competing interests}

The authors wish to declare no known conflicts of interest associated with this study, and there has been no significant financial support for this work that could have influenced the outcome.

\section{Author details}

${ }^{1}$ Department of Physical Medicine and Rehabilitation, National Cheng Kung University Hospital, College of Medicine, National Cheng Kung University, Tainan, Taiwan. ${ }^{2}$ Department of Occupational Therapy, College of Medicine, National Cheng Kung University, Tainan, Taiwan. ${ }^{3}$ Medical Device Innovation Center, National Cheng Kung University, Tainan, Taiwan. ${ }^{4}$ Department of Biomedical Engineering, College of Engineering, National Cheng Kung University, Tainan, Taiwan. ${ }^{5}$ Department of Physical Medicine and Rehabilitation, College of Medicine, National Cheng Kung University, Tainan, Taiwan. ${ }^{6}$ Department of Orthopedics, College of Medicine, National Cheng Kung University, Tainan, Taiwan. ${ }^{7}$ Graduate School of Information Science, Nara Institute of Science and Technology, Ikoma, Japan.

Received: 5 June 2021 Accepted: 4 November 2021 Published online: 23 November 2021

\section{References}

1. Tieland M, Trouwborst I, Clark BC. Skeletal muscle performance and ageing. J Cachexia Sarcopenia Muscle. 2018;9(1):3-19.

2. He H, Luo C, Chang X, Shan Y, Cao W, Gong J, et al. The functional integration in the sensory-motor system predicts aging in healthy older adults. Front Aging Neurosci. 2017;8:306.

3. Kobayashi-Cuya KE, Sakurai R, Sakuma N, Suzuki H, Yasunaga M, Ogawa S, et al. Hand dexterity, not handgrip strength, is associated with executive function in Japanese community-dwelling older adults: a cross-sectional study. BMC Geriatr. 2018;18(1):1-8.

4. Carment L, Abdellatif A, Lafuente-Lafuente C, Pariel S, Maier MA, Belmin J, et al. Manual dexterity and aging: a pilot study disentangling sensorimotor from cognitive decline. Front Neurol. 2018;9:910.

5. Shafizadeh M, Sharifnezhad A, Wheat J. Age-related changes to motor synergies in multi-joint and multi-finger manipulative skills: a metaanalysis. Eur J Appl Physiol. 2019;119(10):2349-62.

6. Chaddock-Heyman L, Loui P, Weng TB, Weisshappel R, McAuley E, Kramer AF. Musical training and brain volume in older adults. Brain Sci. 2021;11(1):50

7. Rosén B, Vikström P, Turner S, McGrouther DA, Selles R, Schreuders T, et al. Enhanced early sensory outcome after nerve repair as a result of immediate post-operative re-learning: a randomized controlled trial. J Hand Surg (European Volume). 2015;40(6):598-606.

8. Serrada I, Hordacre B, Hillier SL. Does sensory retraining improve sensation and sensorimotor function following stroke: a systematic review and meta-analysis. Front Neurosci. 2019;13:402

9. Kim J, Müller K-R, Chung YG, Chung S-C, Park J-Y, Bülthoff HH, et al. Distributed functions of detection and discrimination of vibrotactile stimuli in the hierarchical human somatosensory system. Front Hum Neurosci. 2015:8:1070.

10. Kim M-Y, Kwon H, Yang T-H, Kim K. Vibration alert to the brain: evoked and induced MEG responses to high-frequency Vibrotactile stimuli on the index finger of dominant and non-dominant hand. Front Hum Neurosci. 2020;14:472.

11. Enders LR, Hur P, Johnson MJ, Seo NJ. Remote vibrotactile noise improves light touch sensation in stroke survivors'fingertips via stochastic resonance. J Neuroeng Rehabil. 2013;10(1):1-8.
12. Manjarrez E, Diez-Martınez O, Mendez I, Flores A. Stochastic resonance in human electroencephalographic activity elicited by mechanical tactile stimuli. Neurosci Lett. 2002;324(3):213-6.

13. Sihvonen AJ, Särkämö T, Leo V, Tervaniemi M, Altenmüller E, Soinila S. Music-based interventions in neurological rehabilitation. Lancet Neurol. 2017; 16(8):648-60.

14. Guetin S, Charras K, Berard A, Arbus C, Berthelon P, Blanc F, et al. An overview of the use of music therapy in the context of Alzheimer's disease: a report of a French expert group. Dementia. 2013;12(5):619-34.

15. Thaut $\mathrm{MH}$. The discovery of human auditory-motor entrainment and its role in the development of neurologic music therapy. Prog Brain Res. 2015:217:253-66.

16. Francois C, Grau-Sanchez J, Duarte E, Rodriguez-Fornells A. Musical training as an alternative and effective method for neuro-education and neuro-rehabilitation. Front Psychol. 2015:6:475

17. Tong Y, Forreider B, Sun X, Geng X, Zhang W, Du H, et al. Music-supported therapy (MST) in improving post-stroke patients' upper-limb motor function: a randomised controlled pilot study. Neurol Res. 2015;37(5):434-40.

18. Schneider S, Schönle PW, Altenmüller E, Münte TF. Using musical instruments to improve motor skill recovery following a stroke. J Neurol. 2007;254(10):1339-46.

19. Grau-Sánchez J, Münte TF, Altenmüller E, Duarte E, Rodríguez-Fornells A. Potential benefits of music playing in stroke upper limb motor rehabilitation. Neurosci Biobehav Rev. 2020;112:585-99.

20. Chong HJ, Han SJ, Kim SJ. Keyboard playing as a hand exercise for patients with subacute stroke. Music Ther Perspect. 2017;35(2):144-50.

21. Ripollés P, Rojo N, Grau-Sánchez J, Amengual J, Càmara E, Marco-Pallarés J, et al. Music supported therapy promotes motor plasticity in individuals with chronic stroke. Brain Imaging Behav. 2016;10(4):1289-307.

22. Shu-Ya C, Chun-Fei C. Effects of an active music therapy program on functional fitness in community older adults. J Nurs Res. 2020;28(5):e111.

23. Altenmuller E, Marco-Pallares J, Munte T, Schneider S. Neural reorganization underlies improvement in stroke-induced motor dysfunction by music-supported therapy. Ann N Y Acad Sci. 2009;1169(1):395-405.

24. Friedman N, Chan V, Reinkensmeyer AN, Beroukhim A, Zambrano GJ, Bachman $M$, et al. Retraining and assessing hand movement after stroke using the MusicGlove: comparison with conventional hand therapy and isometric grip training. J Neuroeng Rehabil. 2014;11(1):1-14.

25. Turchet L, West T, Wanderley MM. Touching the audience: musical haptic wearables for augmented and participatory live music performances. Pers Ubiquit Comput. 2021;25(4):749-69.

26. Remache-Vinueza B, Trujillo-León A, Zapata M, Sarmiento-Ortiz F, VidalVerdú F. Audio-tactile rendering: a review on technology and methods to convey musical information through the sense of touch. Sensors. 2021;21(19):6575.

27. Krueger AR, Giannoni P, Shah V, Casadio M, Scheidt RA. Supplemental vibrotactile feedback control of stabilization and reaching actions of the arm using limb state and position error encodings. J Neuroeng Rehabil. 2017;14(1):1-23.

28. Seim C, Doering N, Zhang Y, Stuerzlinger W, Starner T. Passive haptic training to improve speed and performance on a keypad. Proc ACM Interact Mobile Wearable Ubiquitous Technol. 2017:1(3):1-13.

29. Robb SL, Carpenter JS, Burns DS. Reporting guidelines for music-based interventions. J Health Psychol. 2011;16(2):342-52.

30. Chiu HY, Hsu HY, Kuo LC, Chang JH, Su FC. Functional sensibility assessment. Part I: develop a reliable apparatus to assess momentary pinch force control. J Orthop Res. 2009;27(8):1116-21.

31. Hsu HY, Kuo LC, Chiu HY, Jou IM, Su FC. Functional sensibility assessment. Part II: effects of sensory improvement on precise pinch force modulation after transverse carpal tunnel release. J Orthop Res. 2009;27(11):1534-9.

32. Hsu H-Y, Kuo L-C, Kuo Y-L, Chiu H-Y, Jou I-M, Wu P-T, et al. Feasibility of a novel functional sensibility test as an assisted examination for determining precision pinch performance in patients with carpal tunnel syndrome. PLoS One. 2013;8(8):e72064.

33. Hsu H-Y, Kuo L-C, Jou I-M, Chen S-M, Chiu H-Y, Su F-C. Establishment of a proper manual tactile test for hands with sensory deficits. Arch Phys Med Rehabil. 2013;94(3):451-8.

34. Fonseca MCR, Elui VMC, Lalone E, Da Silva NC, Barbosa RI, Marcolino AM, et al. Functional, motor, and sensory assessment instruments upon nerve repair in adult hands: systematic review of psychometric properties. Syst Rev. 2018;7(1):1-11. 
35. Shaffer S, Harrison A, Brown K, Brennan K. Reliability and validity of Semmes-Weinstein monofilament testing in older community-dwelling adults. J Geriatr Phys Ther. 2005;28(3):112-3.

36. Buddenberg LA, Davis C. Test-retest reliability of the Purdue pegboard test. Am J Occup Ther. 2000;54(5):555-8.

37. Flanagan JR, Wing AM. Modulation of grip force with load force during point-to-point arm movements. Exp Brain Res. 1993;95(1):131-43.

38. Flanagan JR, Wing AM. The stability of precision grip forces during cyclic arm movements with a hand-held load. Exp Brain Res. 1990;105(3):455-64.

39. Li K, Wei N, Cheng M, Hou X, Song J. Dynamical coordination of hand intrinsic muscles for precision grip in diabetes mellitus. Sci Rep. 2018;8(1):1-13.

40. Yordanova J, Kolev V, Hohnsbein J, Falkenstein M. Sensorimotor slowing with ageing is mediated by a functional dysregulation of motor-generation processes: evidence from high-resolution event-related potentials. Brain. 2004;127(2):351-62.

41. Franklin DW, Wolpert DM. Computational mechanisms of sensorimotor control. Neuron. 2011;72(3):425-42.

42. Kalisch T, Tegenthoff M, Dinse HR. Improvement of sensorimotor functions in old age by passive sensory stimulation. Clin Interv Aging. 2008;3(4):673

43. Sienko KH, Seidler RD, Carender WJ, Goodworth AD, Whitney SL, Peterka RJ. Potential mechanisms of sensory augmentation systems on human balance control. Front Neurol. 2018;9:944.

44. Mendez-Balbuena I, Manjarrez E, Schulte-Mönting J, Huethe F, Tapia JA, Hepp-Reymond M-C, et al. Improved sensorimotor performance via stochastic resonance. J Neurosci. 2012;32(36):12612-8.

45. Choukou M-A, Mbabaali S, Bani Hani J, Cooke C. Haptic-enabled hand rehabilitation in stroke patients: a scoping review. Appl Sci. 2021:11(8):3712.
46. Halour $\mathrm{MH}$, Sierevelt I, Theuvenet WJ Semmes-weinstein monofilaments: influence of temperature, humidity, and age. J Hand Surg. 2011:36(7):1191-6

47. Saitis $C$, Järveläinen $H$, Fritz $C$. The role of haptic cues in musical instrument quality perception. In: Musical haptics. Cham: Springer; 2018. p. 73-93

48. Seim CE, Wolf SL, Starner TE. Wearable vibrotactile stimulation for upper extremity rehabilitation in chronic stroke: clinical feasibility trial using the VTS glove. J Neuroeng Rehabil. 2021;18(1):1-11.

49. Papetti S, Järveläinen H, Giordano BL, Schiesser S, Fröhlich M. Vibrotactile sensitivity in active touch: effect of pressing force. IEEE Trans Haptics. 2016;10(1):113-22.

50. Zhang Y, Cai J, Zhang Y, Ren T, Zhao M, Zhao Q. Improvement in strokeinduced motor dysfunction by music-supported therapy: a systematic review and meta-analysis. Sci Rep. 2016;6(1):1-8.

51. Trimble M, Hesdorffer D. Music and the brain: the neuroscience of music and musical appreciation. BJPsych Int. 2017;14(2):28-31.

52. Thaut M, Kenyon G, Schauer M, McIntosh $\mathrm{G}$. The connection between rhythmicity and brain function. IEEE Eng Med Biol Mag. 1999;18(2):101-8.

53. Hyde KL, Lerch J, Norton A, Forgeard M, Winner E, Evans AC, et al. Musical training shapes structural brain development. J Neurosci. 2009:29(10):3019-25.

\section{Publisher's Note}

Springer Nature remains neutral with regard to jurisdictional claims in published maps and institutional affiliations.
Ready to submit your research? Choose BMC and benefit from:

- fast, convenient online submission

- thorough peer review by experienced researchers in your field

- rapid publication on acceptance

- support for research data, including large and complex data types

- gold Open Access which fosters wider collaboration and increased citations

- maximum visibility for your research: over $100 \mathrm{M}$ website views per year

At BMC, research is always in progress.

Learn more biomedcentral.com/submissions 